\title{
Restauraciones cerámicas en molares jóvenes con endodoncia
}

\section{Ceramics restorations in young molars with endodontic}

\author{
Urdaneta Quintero $M^{*}$, Yánez de Meléndez L**, Álvarez Zárraga J***, \\ Jimeno Jiménez $M * * * *$, Soto Mestre $C^{* * * * *}$
}

\section{RESUMEN}

Objetivo: Efectuar restauraciones cerámicas Cerec (feldespática y de dióxido de circonio) en molares jóvenes con endodoncia, para prevenir complicaciones en el aparato estomatognático por la pérdida de estos dientes. Material y métodos: Se describe la confección de restauraciones cerámicas a nivel de la zona molar mandibular derecha de un paciente del género femenino de 18 años de edad, a quien se le realizó radiografía periapical, por presentar dolor espontáneo en el lado derecho mandibular. Se diagnosticó una pulpitis irreversible en el primer y segundo molar que fue tratada endodónticamente. Las restauraciones cerámicas de ambos molares fueron confeccionadas con los sistemas CAD/CAM Cerec y evaluadas clínicamente a los 6 y 12 meses, de acuerdo a los criterios establecidos por el Servicio de Salud Pública de los Estados Unidos (USPHS).

Resultados: La evaluación clínica evidenció que las estructuras de cerámica feldespática y de dióxido de circonio ofrecen en el lapso evaluado, adecuada forma anatómica, adaptación marginal, estabilidad en el color, ausencia de caries recidiva a nivel de dientes del sector posterior con tratamiento endodóntico y aceptación del paciente.

Palabras clave: Restauraciones cerámicas, cerámica feldespática, cerámica de dióxido de circonio, molares jóvenes con endodoncia.

\section{SUMMARY}

Purpose: Make a ceramic restoration (feldsphatic and zirconium dioxide) in young molars with endodontic treatment, to prevent further complications in the stomatognathic apparatus for the loss of these teeth.

Material and methods: It describes the making of ceramic restorations in the right mandibular molar area to a female patient of 18 years old, to whom it was made a periapical radiography, for presenting spontaneous pain on the mandibular right side. It was diagnosed as an irreversible pulpitis at the first and second molar, that were treated endodontically. The ceramic restorations of both molars were made with the Cerec CAD/CAM system and were examined after 6 and 12 months in accordance with the US Public Health Service (USPHS) criteria at baseline.

* Docente. Doctora en Odontología, Magister en Odontopediatría. Facultad de Odontología de la Universidad del Zulia. Maracaibo. Venezuela.

** Docente. Doctora en Odontología, Especialista en Prostodoncia y Estética. Facultad de Odontología de la Universidad del Zulia.

*** Alumno de la maestría en Ciencias Dentales. Escuela de Odontología del Recinto de Ciencias Médicas. Universidad de Puerto. Profesor agregado en la Facultad de Odontología de la Universidad del Zulia.

**** Docente. Doctor en Odontología. Especialista en Periodoncia. Facultad de Odontología de la Universidad del Zulia.

***** Odontólogo. Estudiante del postgrado de Docencia Clínica en Odontología. Facultad de Odontología de la Universidad del Zulia. 
Results: The clinical evaluation showed that the structures of feldspathic ceramic and zirconium dioxide in the offer period evaluated presented, proper anatomical shape, marginal adaptation, color stability, absence of recurrence cavities in the posterior teeth with endodontic treatment and acceptance of the patient.

Key words: Ceramic Restorations, feldspathic ceramic, zirconium dioxide ceramic, young molars with endodontic treatment.

Fecha de recepción: 14 de octubre de 2009.

Aceptado para publicación: 4 de noviembre de 2009.

Urdaneta Quintero M, Yánez de Meléndez L, Álvarez Zárraga J, Jimeno Jiménez M, Soto Mestre C. Restauraciones cerámicas en molares jóvenes con endodoncia. Av. Odontoestomatol 2009; 25 (6): 339-343.

\section{INTRODUCCIÓN}

La odontología moderna en la constante búsqueda de biomateriales odontológicos biocompatibles, con adecuadas propiedades mecánicas y resultados estéticos favorables, ha presentado las restauraciones cerámicas de dióxido de circonio sin metal diseñadas y manufacturadas por los sistemas CAD/CAM (diseño asistido por computador/fresado mecánico asistido por computador), como alternativa a las aleaciones metálicas y a los distintos tipos de resinas acrílicas $(1,2)$.

La cerámica de dióxido de circonio en comparación con la cerámica hasta ahora más utilizada de óxido de aluminio, tiene mejores propiedades mecánicas, especialmente en relación con las fuerzas tangenciales y compresivas $(1,3,4)$.

Las propiedades mecánicas y el efecto estético satisfactorio de la cerámica de dióxido de circonio permiten su indicación en coronas unitarias, prótesis fija del sector anterior y posterior y rehabilitaciones en base a implantes dentales $(1,5,6$,). Para la elaboración de incrustaciones y carillas se han recomendado las porcelanas feldespáticas porque hacen posible realizar restauraciones conservadoras con resistencia y estética (7).

Las coronas cerámicas de dióxido de circonio empleadas en molares tratados endodónticamente con pernos de fibra de vidrio, han demostrado alta resis- tencia a la fractura (8). Asimismo constituyen una alternativa de tratamiento para pacientes con bruxismo y maloclusión gracias a sus buenas propiedades mecánicas $(9,10)$.

El propósito del presente artículo fue efectuar restauraciones cerámicas Cerec (feldespática y de dióxido de circonio) en molares jóvenes tratados endodónticamente, para prevenir complicaciones en el aparato estomatognático por la perdida de estos dientes.

\section{MATERIAL Y MÉTODOS}

Se presenta el caso de un paciente del género femenino de 18 años de edad, quien acude a la consulta de la Facultad de Odontología de la Universidad del Zulia, Maracaibo, Venezuela por presentar dolor espontáneo a nivel mandibular del lado derecho.

Al realizar la evaluación clínica y radiográfica se diagnosticó apiñamiento dental del sector anterior maxilar y mandibular, además de pulpitis irreversible a nivel del primer molar mandibular del lado derecho (46) y del segundo molar (47), por lo cual se realizó tratamiento endodóntico. Previo consentimiento del paciente se planificó restaurar ambos molares con restauraciones cerámicas confeccionadas con los sistemas CAD/CAM Cerec ${ }^{\circledR}$ in lab (Sirona). A continuación se explica el procedimiento empleado. 
Se realizó gingivectomía y osteotomía a nivel del diente 46 para alargamiento coronal y realizar restauración con corona perno. Finalizado el tiempo de cicatrización se desobturó el conducto distal, dejando 5 $\mathrm{mm}$ de gutapercha a nivel apical, luego se cemento un perno de fibra de vidrio de 1,5 mm Reforpost RX (Angelus ${ }^{\circledR}$, Brasil) utilizando cemento resinoso dual RelyX ${ }^{\mathrm{TM}}$ Unicem (3M Espe). Posteriormente se reconstruyó la corona del diente con la resina compuesta fotocurada Filtek $^{\mathrm{TM}} \mathrm{Z100}^{\mathrm{TM}}$ (3M Espe).

Para el tallado del muñón se realizó preparación con hombro recto y ángulo interior redondeado empleando fresas diamantadas cilíndricas de extremo redondeado. Margen epigingival con uniforme trayectoria circular de la preparación, eliminando 0,6 mm de tejido dental circular y $1,5 \mathrm{~mm}$ de reducción oclusal (1) (Fig. 1). Se confeccionó la corona provisional con la técnica directa de fabricación de provisionales empleando acrílico Alike (GC América), utilizando una matriz preformada obtenida de un encerado diagnóstico previo.

Se efectuó tallado de cavidad Oclusodistal (OD) a nivel del 47 para la confección de una incrustación. Se utilizó piedras de diamante (Kit de la casa KG Sorensen) preparando paredes rectas, uniformes y planas con ángulos axiopulpares redondeados (11). Nivelando piso de la cámara pulpar con ionómero de vidrio fotopolimerizable Ionoseal de la casa Voco (Fig. 1).

La retracción gingival a nivel del 46 fue lograda mediante el hilo retractor Ultrapak \# 00 (Ultradent) impregnado con astringente hemostático ViscoStat clear (Ultradent). Para la toma de impresión se usó la técnica de la doble impresión con la silicona por adición en consistencia de masilla y liviana President (Coltene/ Whaledent). La impresión fue realizada en un solo tiempo.

Para la confección del núcleo de la corona se usó un bloque de circonio C15 diseñado con el sistema CAD/ CAM Cerec ${ }^{\circledR}$ in lab (Sirona). Fresada en la máquina Inlab sinterizando por 7 horas en el ZYcomat, máquina de sinterización del circonio. El núcleo fue recubierto con cerámica para circonio VM9 de Vita (Vita Zahnfabrik). La incrustación fue realizada empleando cerámica feldespática.

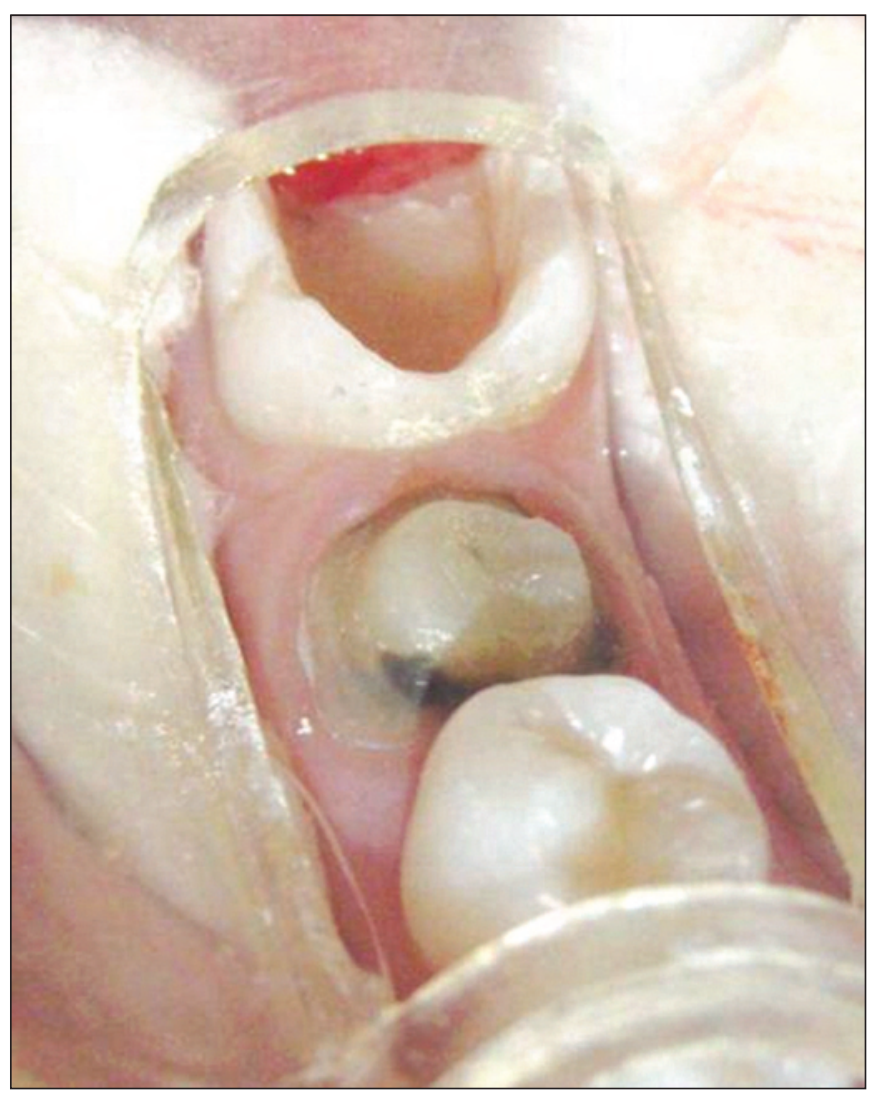

Fig. 1. Tallado del muñón (46) y preparación cavitaria para la incrustación (47).

Confeccionadas las restauraciones se precedió a probarlas, adaptarlas y cementarlas definitivamente con el cemento adhesivo Panavia 21 (Kuraray dental, Osaka, Japón) efectuando control de oclusión (Fig. 2).

Las restauraciones cerámicas de ambos molares fueron evaluadas clínicamente a los 6 y 12 meses por un operador previamente calibrado de acuerdo a los criterios establecidos por el Servicio de Salud Pública de los Estados Unidos (USPHS) (12). Asimismo se efectuó control radiográfico (Fig. 3).

\section{RESULTADOS}

La evaluación clínica efectuada a los 6 y 12 meses evidenció que las estructuras de cerámica feldespática y de dióxido de circonio ofrecen un comportamiento positivo relacionado con la categoría alfa en 


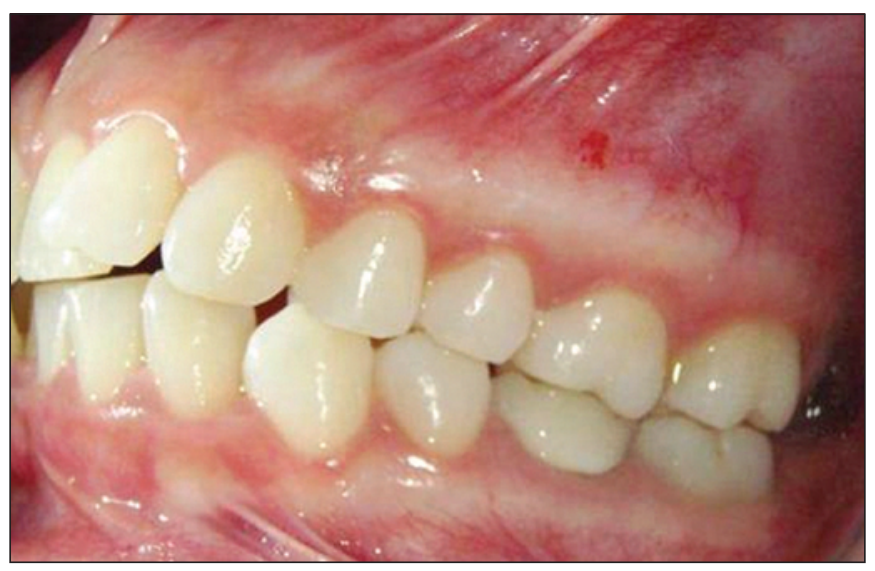

Fig. 2. Cementación definitiva.

cuanto a forma anatómica y adaptación marginal, igualmente se observó estabilidad en el color indicada por la categoría bravo y ausencia de caries recidiva representada por la categoría alfa. El control radiográfico no reveló patología existente.

\section{DISCUSIÓN}

Se ha planteado que la calidad de las restauraciones diseñadas y manufacturadas con el sistema CAD/ CAM no iguala a la obtenida por el procedimiento de laboratorio convencional. $(11,13)$. Sin embargo el método computarizado ha demostrado ser altamente eficaz para confeccionar restauraciones cerámicas de excelente calidad clínica.

El comportamiento positivo de las restauraciones cerámicas (feldespática y de dióxido de circonio)

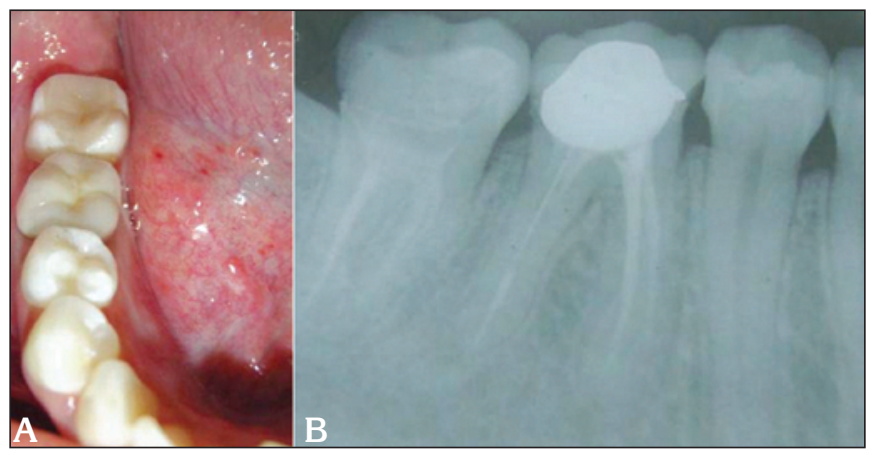

Fig. 3. A: Evaluación clínica. B: Evaluación radiográfica. evaluado en el lapso de un año demuestra la confiabilidad clínica de las estructuras confeccionadas por los sistemas CAD/CAM Cerec. Asimismo Mormann y Krejci, 1992 (14) comprobaron que las incrustaciones cerámicas adhesivas (Cerec) pueden alcanzar un periodo de 5 años con éxito clínico.

Isenberg, Essig y Leinfelder, 1992 (15) observaron la permanencia del color e integridad marginal de las incrustaciones evaluadas durante un periodo de 3 años. En un estudio clínico de 50 incrustaciones (Cerec) llevada a cabo por Heymann y cols, 1996 (16) se comprobó que la pérdida de cemento en el margen de la restauración era baja desde el tercer al cuarto año.

Wrbas y cols, 2007 (17) al evaluar clínicamente las incrustaciones cerámicas (Cerec) durante un periodo de 2 años encontraron que no había cambios significativos en el color , superficie y forma anatómica de las restauraciones.

Otto y Schneider, 2008 (7) concluyeron mediante un estudio de 15 años que las restauraciones de cerámica feldespática (Cerec) presentan vida útil en la cavidad bucal a largo plazo.

Atendiendo a estas consideraciones se ha incrementado el uso de estructuras cerámicas libres de metal para la restauración de dientes con tratamiento de conducto y severa destrucción coronal. Así como también sometidos a fuertes cargas masticatorias por problemas de mal posición dental (8-10).

Finalmente consideramos que las restauraciones de cerámica feldespática y dióxido de circonio diseñadas y confeccionadas con el sistema CAD/CAM Cerec ofrecen una alternativa de tratamiento para pacientes con molares tratados endodónticamente.

\section{BIBLIOGRAFÍA}

1. Kurbad A, Reichel K. Restauraciones CAD/CAM de cerámica de óxido de circonio sin metal. Quintessence. 2006;19(8):458-69

2. Manicone PF, Rossi IP, Raffaelli L, Paolantonio M, Rossi G, Berardi D, Perfetti G. Biological 
considerations on the use of circonio for dental devices. International Journal of Immunopathology and Pharmacology. 2007;20:9-12.

3. Sadan A, Blatz M, Lang B. Clinical considerations for densely sintered alumina and zirconia restorations: Part 1. International Journal Periodontic Restorative Dentistry. 2005;25(3): 213-9.

4. Vult von Stevern P, Ebbesson S, Holmgren J, Haag P, Nilner K. Fracture strength of two oxide ceramic crown systems after cyclic preloading and thermocycling. Journal of Oral Rehabilitation. 2006; 33(9):682-9.

5. Manicone PF, Rossi IP, Raffaelli L. An overview of zirconia ceramic : basic properties and clinical applications. Journal of Dentistry. 2007;35(11): 819-25.

6. Rosentritt M, Behr M, Rinke S, Handel G. Cerámica de óxido de circonio en prótesis de coronas y puentes. Quintessence. 2008;21(1):23-30.

7. Otto T, Schneider D. Long term clinical results of chairside Cerec CAD/CAM inlays and onlays: a case series. International Journal of Prosthodontic. 2008;21(1):53-9.

8. Salameh Z, Ounsi HF, Aboushelib MN, Sadig W, Ferrari $M$. Fracture resistance and failure patterns of endodontically treated mandibular molars with and without glass fiber post in combination with a zirconia ceramic crown. Journal of Dentistry. 2008;36(7):513-9.

9. Kessler- Liechti G, Mericske-Stern R. Rehabilitation of an abraded occlusion with Procera$\mathrm{ZrO} 2$ all ceramic crowns. A case report. Schweizer Moatsschrift fur Zahnmedizin. 2006; 116(2):15667.

10. Toksavul S, Turkun M, Toman M. Esthetic enhancement of ceramic crowns with zirconia dowels and cores: a clinical report. The Journal of Prosthetic Dentistry. 2004; 92(2):116-9.
11. Goldstein R. Odontología Estética. Principios, comunicación y métodos terapéuticos. Vol. 1. Editorial Ars. Médica: Barcelona España; 2002. p:395-98.

12. Cvar J, Ryge G. Criteria for the clinical evaluation of dental restorative materials. U.S. Public Health Service Publication Na 709-244. San Francisco, U.S. Government Printing Office; 1971.

13. Srinivasan M. Un conventional prosthodontics: Post, core and crown technique. The Journal of Indian Prosthodontic Society. 2007;7(4):19195.

14. Mormann W, Krejci I. Computer-designed inlays after five years in-situ: clinical performance and scanning electron microscopic evaluation. Quintessence International. 1992;23:109-15.

15. Isenberg B, Essig M, Leinfelder K. Theree-year clinical evaluation of CAD/CAM restoration. Journal of Esthetic Dentistry 1992;4(5):173-6.

16. Heymann H, Bayne S, Sturdevant J, Wilder A, Roberson T. The clinical performance of CAD/ CAM generated ceramic inlays: a four year study. Journal American Dental Association. 1996; 127(8):1171-81.

17. Wrbas KT, Hein N, Schirrmeister JF, Altenburger MJ, Hellwig E. Two year clinical evaluation of Cerec 3D ceramic inlays inserted by undergraduate dental students. Quintessence International. 2007;38(7):575-81.

\section{CORRESPONDENCIA}

M. Urdancia Quintero

Facultad de Odontología de la Universidad del Zulia. Calle 65. Av 19.

Edificio Ciencia y Salud. Tercer piso.

División de Estudios para Graduados.

Maracaibo. Venezuela.

Correo electrónico: miliur69@hotmail.com 